\section{Internato rural em odontologia no Rio de Janeiro, Brasil}

\author{
Branca Heloísa de Oliveira ${ }^{1}$
}

\section{RESUMO}

Este trabalho descreve a experiência da Faculdade de Odontologia da Universidade do Estado do Rio de Janeiro com o internato rural, no período de 1988 a 1993, como parte do processo de sensibilização social do aluno de graduação. As atividades extramurais foram realizadas por meio de estágios supervisionados de 3 meses consecutivos nos serviços odontológicos de unidades de saúde da rede pública, em municípios do interior do estado do Rio de Janeiro. Além de executarem atendimento clínico, os estudantes participaram de atividades cujos temas se relacionavam a: situação de saúde bucal da comunidade; estrutura organizacional e programática das secretarias de saúde; e prioridades, tecnologia apropriada, educação e prevenção em saúde bucal. A modalidade de educação apresentada resultou integradora da reflexão crítica de docentes, alunos, associações de classe e entidades prestadoras de serviços acerca da adequação do profissional formado nos cursos de graduação em odontologia no Rio de Janeiro à realidade do estado.

\footnotetext{
1 Universidade do Estado do Rio de Janeiro, Faculdade de Odontologia. Correspondência e pedidos de separatas devem ser enviados para o seguinte endereço: Faculdade de Odontologia, UERJ, Bvd. Vinte e Oito de Setembro 157, Vila Isabel, CEP 20551-030, Rio de Janeiro, RJ, Brasil. Email: brancaheloisa@ibm.net
}

Embora o Brasil disponha de considerável número de cirurgiões-dentistas, tecnicamente bem qualificados, pouco se tem feito, em nível nacional, para melhorar as condições de saúde bucal da população. O relatório final da $1^{\mathrm{a}}$ Conferência Nacional de Saúde Bucal (1), realizada em 1986, afirma que a situação de saúde bucal no país é caótica e que o modelo de prática odontológica cobre as necessidades de apenas 5\% da população.

O levantamento epidemiológico realizado no mesmo ano pelo Ministério da Saúde (2) confirma esta afirmação, ao revelar altos índices de cárie dental entre as crianças brasileiras, as quais têm uma das mais altas médias de dentes cariados, perdidos e obturados (CPO) do mundo. Além disso, o levantamento evidencia a elevada prevalência de edentulismo na idade adulta, provocada pela ausência de tratamento adequado ao longo da vida.

Não se pode ignorar, como assinalou Uchôa (3), que a crise na assistência odontológica não ocorre de maneira isolada. Está ligada à crise econômica, social e política do país, associada à distorção do sistema formador de recursos humanos que prepara profissionais para atuação em clínica privada. A análise da formação profissional na área da saúde no Brasil revela, também, a predominância de planos de estudo voltados à formação baseada no domínio psicomotor e, secundariamente, no cognitivo, desconsiderando o campo afetivo. Como conseqüência, segundo Garrafa (4), profissionais com este treinamento carecem de senso crítico e criatividade para responder de modo objetivo e integrado aos desafios na área da saúde de um país em crise. Por este motivo, os egressos dos cursos de odontologia são, muitas vezes, vistos como técnicos preparados unicamente para tratar as doenças, incapazes de refletir sobre sua prática social ou de reconhecer o complexo bio-psicossocial do indivíduo (5).

Levando essas questões em consideração, as entidades envolvidas com a formação de recursos humanos em odontologia no país propuseram um novo currículo mínimo para os cursos de graduação, aprovado em 1982 pelo Conselho Federal de Educação $(6,7)$. Esse currículo tem por objetivo formar cirurgiões-dentistas com uma perspectiva preventivo-social da prática odontológica, com habilidade para responder às demandas de atenção buco-dental da população onde esteja localizada a instituição de ensino e aptos para o trabalho em equipe. Propõe a adoção de um plano de ensino 
orientado à prevenção, baseado em conceitos de sociologia, antropologia e psicologia, com a execução de atividades junto ao paciente, antecipadas e desenvolvidas em estágios supervisionados, de preferência em sistemas públicos de saúde.

A Faculdade de Odontologia da Universidade do Estado do Rio de Janeiro (UERJ) (8) ajustou-se à modificação do currículo mínimo, mediante mudanças em seu currículo pleno, conforme a deliberação 107/84, de 27 de abril de 1984. Dentre outros pontos, esta deliberação estabeleceu que conhecimentos de sociologia, antropologia e psicologia seriam incorporados à disciplina de odontologia social e que o oitavo período do curso de graduação seria dedicado exclusivamente às atividades extramurais, com 450 horas de aula. O objetivo dessas atividades era "... desenvolver o aprendizado clínico global (atendimento restaurador, preventivo e cirúrgico), assim como as atividades de educação em saúde, através de estágios supervisionados nas áreas hospitalar, ambulatorial, programas de atendimento à comunidade, ao excepcional, serviços odontológicos escolares, além de proporcionar experiência nas áreas de organização e administração de serviços de saúde" (Catálogo do Centro Biomédico/UERJ, 1987:68).

$\mathrm{Na}$ realidade, as atividades extramurais já vinham sendo desenvolvidas sob a forma de estágio curricular desde 1982 na Faculdade de Odontologia da UERJ, sob orientação dos professores de odontologia social. Naquele ano, implantou-se um programa docente-assistencial (9), por meio de convênio entre a Universidade e o Ministério de Educação e Cultura, ${ }^{2}$ com a construção de uma clínica odontológica simplificada no Colégio de Aplicação da UERJ, tendo essa experiência se estendido, mais tarde, às escolas da rede municipal de ensino do Rio de Janeiro.

Porém, não tardou para que os próprios docentes envolvidos com as atividades extramurais percebessem que a mudança curricular, embora proporcionasse o aumento da carga horária destinada a estas atividades, não seria suficiente, como bem havia ressaltado a ABENO (10), para estimular o desenvolvimento da sensibilidade social dos estudantes. Medeiros (11) demonstrou, por exemplo, que apenas $30 \%$ dos alunos do último período da Faculdade de Odontologia da UERJ poderiam ser considerados socialmente sensíveis e que as atividades extramurais, da forma como vinham sendo executadas, não possibilitavam uma mudança na percepção dos demais a esse respeito. Além disso, verificou que, dos 30 estudantes entrevistados em

\footnotetext{
2 Através da Fundação Coordenação de Aperfeiçoamento de Pessoal de Nível Superior (CAPES), da Associação Brasileira de Ensino Odontológico (ABENO) e da Fundação Kellog.
}

sua pesquisa, seis afirmaram que a atividade não era importante, pois só permitia a prática de odontologia clínica e de cirurgia, temas já amplamente estudados durante o curso. Esse posicionamento evidencia, de acordo com Medeiros (11), uma preocupação quase que exclusiva desses alunos com o aprendizado de técnicas de tratamento clínico.

Orientada por esta percepção, a direção da Faculdade de Odontologia promoveu, em 1988, o I Seminário Integrado sobre Ensino Odontológico, com o propósito de integrar o aparelho formador de recursos humanos, o subsistema prestador de serviços e as entidades de classe para debaterem questões relativas à saúde bucal no estado do Rio de Janeiro e estabelecerem o grau de resolutividade do ensino de gradução em odontologia. Esse encontro concluiu que "o sistema educacional não vinha atendendo satisfatoriamente à formação de profissionais, nem para o setor privado, nem para o setor público, na medida em que o recém-formado não se sentia preparado com segurança, mesmo nas tarefas consideradas básicas na clínica geral, procurando os cursos de especialização para melhor se capacitar". Entre as sugestões apresentadas para reverter este quadro, destacam-se as propostas de complementar o ensino com educação em ciências sociais, prática em programas extramurais, formação preferencial de clínicos gerais e reflexão sobre as realidades de ensino e serviço (12).

A partir das conclusões do seminário, desenvolveu-se um ambiente propício para a Faculdade incorporar a experiência do internato rural, desenvolvida até então com alunos do curso de medicina pelo Instituto de Medicina Social da UERJ. Os alunos de odontologia teriam oportunidade de apreender a realidade social que os cercava, através de estágios em unidades da rede pública de saúde, situadas em municípios do interior do próprio estado em que estavam sendo formados e não apenas em clínicas planejadas, implantadas e administradas pelos professores. Atendia-se, também, com maior rigor, às proposições do currículo mínimo, já mencionado anteriormente.

\section{INTERNATO RURAL EM ODONTOLOGIA}

As atividades do internato rural em odontologia foram iniciadas nos municípios de Natividade e São Fidélis, estado do Rio de Janeiro, no segundo semestre letivo de 1988, com o objetivo de oferecer aos alunos e docentes a oportunidade de 1) compreender o desenvolvimento do processo saúdedoença no contexto socioeconômico e cultural das comunidades trabalhadas; 2 ) identificar o grau de resolutividade da rede de serviços odontológicos e a forma de melhorar a prática assistencial, princi- 
palmente no setor público, e, ao mesmo tempo, promover a retroalimentação do processo de ensinoaprendizagem; 3) participar de uma equipe multiprofissional de saúde; e 4) redefinir o papel da Faculdade de Odontologia da UERJ dentro do processo de inserção do sistema formador de recursos humanos na implantação do Sistema Único de Saúde no estado do Rio de Janeiro (13).

O funcionamento do internato rural foi possível pela assinatura de convênios de cooperação técnico-científica entre a Universidade e as prefeituras interessadas no projeto. Para alcançar os objetivos acima mencionados, os alunos do oitavo período do curso de graduação, após optarem pelo internato rural, eram encaminhados, em grupos de no mínimo dois por município, aos coordenadores de odontologia das secretarias municipais de saúde. Os estudantes permaneciam residindo por 3 meses consecutivos nos locais de estágio, cumprindo uma jornada diária de 8 horas em atividades relacionadas com os seguintes tópicos (13):

- estrutura organizacional da secretaria municipal de saúde;

- programas de saúde e rede de serviços existente;

- composição da equipe de saúde, funções e integração de seus membros;

- problemas de saúde bucal dos diferentes grupos populacionais do município;

- prioridades definidas para o tratamento odontológico;

- processo de trabalho de educação em saúde da população;

- importância da utilização de tecnologia apropriada;

- vantagens do emprego de pessoal auxiliar;

- necessidade do emprego de métodos preventivos para grandes populações;

- soluções encontradas pelas comunidades para os problemas de saúde bucal.

A primeira semana de estágio era dedicada ao conhecimento do campo pelo aluno, acompanhado pelo coordenador municipal de odontologia, à distribuição de tarefas e à escolha de temas para os grupos de estudo quinzenais, dos quais participavam todos os membros da equipe odontológica. Durante as semanas seguintes, os alunos continuavam seu trabalho em serviços odontológicos mantidos pelo município, supervisionados pelo coordenador municipal de odontologia e recebendo visitas periódicas, semanais ou quinzenais, de acordo com a necessidade, de professores da Faculdade de Odontologia. Deve-se ressaltar que a supervisão efetuada pelo coordenador local tinha como objetivo principal apoiar, acompanhar e observar o estagiário, diferindo bastante daquela feita nas clí- nicas mantidas intramuros pela Faculdade de Odontologia, onde o aluno costuma trabalhar sob a supervisão direta de um professor, que avalia e autoriza todos os passos dos procedimentos odontológicos realizados. No internato rural, a intervenção do supervisor era mínima, para que o futuro profissional pudesse exercitar plenamente sua capacidade de decisão, baseado no que aprendeu durante o curso de graduação.

A avaliação dos estagiários era feita de modo contínuo, pelas equipes da secretaria municipal de saúde e da Faculdade de Odontologia. Ao final do período, o aluno era solicitado a apresentar um relatório final, uma auto-avaliação e a avaliação de seu supervisor. Para a elaboração do relatório final, recebia um formulário onde estavam relacionados os principais pontos a serem abordados, como por exemplo, situação geográfica do município onde havia realizado o estágio; indicadores socioeconômicos da população; principais problemas de saúde identificados; tecnologias empregadas em nível local para a sua solução; e sugestões para o aperfeiçoamento dos serviços odontológicos. A autoavaliação era feita com base nos seguintes critérios: assiduidade, pontualidade; relacionamento com os pacientes, o supervisor e os demais membros da equipe; respeito às orientações dos professores; domínio técnico; iniciativa no trabalho; criatividade na superação das dificuldades; receptividade a críticas e sugestões; participação ativa na vida da comunidade; e apresentação pessoal. A avaliação do supervisor era efetuada pelos estagiários conforme os seguintes itens: precisão das orientações recebidas; freqüência de comparecimento aos locais de estágio; rapidez na solução dos problemas; receptividade a críticas e sugestões; preocupação com o estímulo ao espírito de equipe e à integração dos estudantes com a comunidade.

\section{CONCLUSÕES}

Entre agosto de 1988 e maio de 1993 participaram do internato rural em odontologia 100 alunos (12) do oitavo período do curso de graduação. Os relatórios apresentados pelos mesmos permitem afirmar que o estágio produziu resultados favoráveis, tanto do ponto de vista de sua formação profissional quanto pessoal. Os aspectos positivos destacados com maior freqüência pelos estudantes compreenderam:

- aquisição de agilidade no trabalho devido à grande demanda da população para tratamento;

- desenvolvimento de autoconfiança e senso crítico, devido à oportunidade de testar os conhecimentos adquiridos durante o curso, ao ter que 
tomar decisões sem o auxílio constante de um professor;

- aprimoramento de relações pessoais, tanto no contato com outros colegas quanto com os pacientes, dentro e fora do ambiente de trabalho;

- intercâmbio de informações com estagiários de outras profissões de saúde (medicina, nutrição e enfermagem);

- apreensão mais precisa das reais condições de vida da população fluminense, através do contato com uma realidade socioeconômica e cultural diferente daquela do aluno;

- conscientização acerca de seu papel na sociedade enquanto cidadão e cirurgião-dentista, ao verificar quanto pode ser útil à comunidade, através do exercício sério e competente de sua profissão.

Do ponto de vista dos municípios, o internato rural também apresentou efeitos benéficos, ao introduzir modificações filosóficas e metodológicas, que se refletiram no tipo de assistência às populações atingidas pelo programa. Em Natividade, por exemplo, no ano de 1987, antes da implantação do internato rural, efetuaram-se, nos serviços odontológicos da secretaria municipal de saúde, 6703 exodontias, sem sequer um tratamento odontológico completo. Em 1990 (até outubro), efetuaram-se apenas 705 exodontias e 529 pacientes tiveram seus tratamentos completados nos mesmos serviços. Percebe-se, assim, uma nítida mudança no enfoque dado à odontologia, possível não apenas devido ao trabalho dos alunos da Faculdade de Odontologia da UERJ, como também à assessoria ao município prestada pelo corpo docente da Faculdade, que desenvolveu, entre outras ativida- des, um curso de formação de técnico em higiene dental (14).

A possibilidade de interação do corpo docente da Faculdade nas atividades promovidas pelo internato rural pode ademais servir, segundo Uchôa (13), como instrumento de retroalimentação para o próprio ensino de graduação, pelo fato de:

- estimular os docentes a comparar o conteúdo de suas disciplinas com as necessidades da população, observadas no campo de estágio;

- facilitar a capacitação de professores no manejo de metodologia para integrar a teoria à prática;

- favorecer a reflexão sobre a prática pessoal e coletiva, para identificar indicadores de caminhos a percorrer, com a finalidade de oferecer serviços odontológicos adequados à coletividade.

Finalmente, deve-se registrar que a experiência aqui relatada não constitui um modelo de estágio pronto e acabado, mas uma proposição de caráter dinâmico, sempre reformulada de acordo com as observações feitas pelos docentes, alunos e profissionais das secretarias municipais de saúde e pelas populações assistidas por meio do programa.

Agradecimento. A autora agradece a oportunidade de participar da experiência pedagógica de implantação do internato rural na Faculdade de Odontologia da UERJ e reconhece a iniciativa e obstinação do Professor Hélio Uchôa, da disciplina de odontologia social, fundamentais para a garantia da continuidade dessa experiência ao longo de todo o tempo em que a mesma foi desenvolvida.

\section{REFERÊNCIAS}

1. Brasil, Ministério da Saúde, Universidade Nacional de Brasília. Relatório Final, I Conferência Nacional de Saúde Bucal 1986:2-6.

2. Brasil, Ministério da Saúde. Levantamento epidemiológico em saúde bucal: Brasil, zona urbana, 1986. Brasília: Centro de Documentação do Ministério da Saúde; 1988.

3. Uchôa HW. Assistência odontológica no país: perspectivas 2. Cad Saude Publica 1985;1:263-269.

4. Garrafa V. Contra o monopólio da saúde. Rio de Janeiro: Achiamé; 1983.

5. Paixão HH, Campos H, Lima HA. O paciente como objeto de ensino. Arch Cent Estud Fac Odont UFMG 1981;18: $37-44$.
6. Brasil, Conselho Federal de Educação. Parecer 370/82. Documenta 1982;260: 46-54.

7. Brasil, Poder Executivo, Resolução 04/82. Diário Oficial da União. Brasília: 16 de setembro de 1982 .

8. Universidade do Estado do Rio de Janeiro. Deliberação 107/84. Rio de Janeiro: 27 de abril de 1984.

9. Universidade do Estado do Rio de Janeiro, Faculdade de Odontologia. Projeto docente-assistencial; 1982. (Documento mimeografado).

10. Associação Brasileira de Ensino Odontológico. Integração dos ciclos básico e profissional nos cursos de Odontologia: conclusões do tema oficial, XXII Reunião. Camaragibe; 1986.
11. Medeiros UV. A experiência extramural como resposta às necessidades de saúde bucal da comunidade [tese de livre docência]. Rio de Janeiro: Universidade do Estado do Rio de Janeiro; 1987.

12. Universidade do Estado do Rio de Janeiro, Faculdade de Odontologia. Seminário Integrado sobre Ensino Superior, 1: relatório final. Rio de Janeiro; 1988.

13. Uchôa HW. Internato rural: odontologia. Faculdade de Odontologia/UERJ Rio de Janeiro; 1992. (Documento mimeografado).

14. Kabouk IMB. Evolução da odontologia no município de Natividade, Rio de Janeiro, 1987/90, aspectos gerais. Natividade; 1990. (Documento mimeografado). 
ABSTRACT This paper describes a rural internship experience developed between 1988 and 1993 by the School of Dentistry, Rio de Janeiro State University. The objective of this program was to raise the social consciousness of under-

Rural internship in dentistry in Rio de Janeiro, Brazil graduate students. Extramural activities were carried out in the form of supervised 3-month internships in the dentistry services offered by public health units at several locations in the state of Rio de Janeiro. Besides working in the clinics, students took part in activities aimed at assessing oral health in the community, organizational and programmatic structures of the health services, and priorities, appropriate technology, education, and prevention in oral health. These internships integrated the critical thinking of professors, students, class associations, and service-providing institutions regarding the adequacy of education received by future dentists in addressing the true situation of oral health in the state of Rio de Janeiro.

\section{Taller Latinoamericano sobre la Reforma del Sector Salud}

Fechas: 18 al 23 de octubre de 1998

Lugar: San Cristóbal de las Casas, Chiapas, México

Organiza este segundo taller el Colegio de la Frontera Sur (ECOSUR) con los auspicios del Consejo Británico, la Organización Panamericana de la Salud, la Escuela de Medicina Tropical de la Universidad de Liverpool, el Ministerio de Salud de México y el Instituto Mexicano del Seguro Social. En el taller se tratarán los siguientes temas: políticas y principios de la reforma, globalización y acción local, estrategias y mecanismos de acción intersectorial, modelos de organización, descentralización, atención a grupos y áreas más necesitados, participación social, potencial humano en la reforma, financiamiento y evaluación del proceso de reforma. Pueden obtenerse detalles y formularios de preinscripción en las direcciones que siguen.

Información:

Dr. Héctor Ochoa, Coordinador, II TLARES

ECOSUR, Carretera Panamericana y Periférico Sur s/n

Barrio de Ma. Auxiliadora, C.P. 29290

San Cristóbal de las Casas, Chiapas, México

Teléfono: (+52-967) 81883, ext. 2001 y 2002

Fax: (+52-967) 82322

Correo electrónico: tlars@sclc.ecosur.ms

0 bien

hochoa@sclc.ecosur.mx

Internet: http://www.ecosur.mx/eventos/tlares.htm 